\title{
GENERAL SOLUTION OF NONLINEAR DIFFERENCE EQUATIONS
}

\author{
W. A. HARRIS, JR. ${ }^{\text {BY }}{ }^{\text {) }}$ AND Y. SIBUYA $\left({ }^{2}\right)$
}

1. Introduction. We present a method for solving a system of nonlinear difference equations of the form

$$
y(x+1)=f(x, y(x)) \text {, }
$$

where $x$ is a complex variable, $y$ is an $n$-dimensional vector, and $f(x, y)$ is an $n$-dimensional vector with components holomorphic in the region

$$
\operatorname{Im}(x) \geqq R_{0}, \quad\|y\|=\underset{i=1}{n}\left|y_{i}\right| \leqq \delta_{0},
$$

the $y_{i}$ being components of $y$. We assume that $f$ admits a uniformly asymptotic expansion

$$
f(x, y) \cong \sum_{k=0}^{\infty} x^{-k} f_{k}(y)
$$

for $\|y\| \leqq \delta_{0}$ as $x$ tends to infinity in the region $\operatorname{Im}(x) \geqq R_{0}$. The coefficients $f_{k}(y)$ are assumed to be holomorphic for $\|y\| \leqq \delta_{0}$. Let $\lambda_{i}$ be the eigenvalues of the matrix $f_{0 y}(0)$. We shall make the following assumptions:

(i) $f_{0}(0)=0$,

(ii) $1<\left|\lambda_{1}\right|<\left|\lambda_{2}\right|<\cdots<\left|\lambda_{n}\right|$,

(iii) $\prod_{i=1}^{n}\left|\lambda_{i}\right|^{p_{i}} \neq\left|\lambda_{j}\right|$

for $j=1,2, \cdots, n$ and $\sum_{i=1}^{n} p_{i} \geqq 2$, where $p_{i}$ are nonnegative integers.

If $f_{0}(0)=0$ and $\lambda_{i} \neq 1$, we can determine a formal solution

$$
y=\sum_{k=1}^{\infty} x^{-k} p_{k}
$$

of the system of difference equation (1.1) by substitution and identification of terms. In one of our previous papers [3] we have shown that, if $f_{0}(0)=0$ and $\left|\lambda_{i}\right| \neq 1$, there exists a holomorphic solution $y=p(x)$ of the system (1.1) in the region

$$
\operatorname{Im}(x) \geqq R_{1} \quad\left(>R_{0}\right)
$$

such that $p(x)$ admits the formal solution (1.2) as asymptotic expansion as $x$ tends to infinity in the region (1.3).

Presented to the Society, January 25, 1964; received by the editors December 11, 1963.

(1) Supported in part by the National Science Foundation under Grant G-18918.

(2) Supported in part by a Contract of the Office of Naval Research, Nonr-3776(00). 
By the transformation

$$
y=u+p(x)
$$

the system (1.1) is reduced to a system of the form

$$
u(x+1)=g(x, u(x)),
$$

where

$$
g(x, u)=f(x, u+p(x))-p(x+1) .
$$

The quantity $g(x, u)$ is holomorphic for (1.3) and $\|u\| \leqq \delta^{\prime}$, and admits a uniformly asymptotic expansion

$$
g(x, u) \cong \sum_{k=0}^{\infty} x^{-k} g_{k}(u)
$$

for $\|u\| \leqq \delta^{\prime}$ as $x$ tends to infinity in the region (1.3). It is easily seen that

$$
g_{0}(u)=f_{0}(u) .
$$

On the other hand, since $g(x, 0)=0$, we can write $g$ as

$$
g(x, u)=B(x) u+\sum_{|\mathfrak{p}| \geqq 2} \hat{g}_{\mathfrak{p}}(x) u^{\mathfrak{p}},
$$

where $\mathfrak{p}$ is a set of nonnegative integers $p_{1}, \cdots, p_{n}$ and $u^{\mathfrak{p}}=u_{1}^{p_{1}} \cdots u_{n}^{p_{n}},|\mathfrak{p}|$ $=p_{1}+p_{2}+\cdots+p_{n}$. The coefficients $B(x)$ and $g_{p}(x)$ are holomorphic for (1.3) and admit asymptotic expansions

$$
\begin{aligned}
& B(x) \cong \sum_{k=0}^{\infty} x^{-k} B_{k}, \\
& g_{p}(x) \cong \sum_{k=0}^{\infty} x^{-k} g_{\mathfrak{p} k},
\end{aligned}
$$

as $x$ tends to infinity through the region (1.3). Furthermore,

$$
B_{0}=g_{0 y}(0)=f_{0 y}(0) .
$$

Due to a result which we have proved in one of our previous papers [4], we may assume without loss of generality that $B(x)$ is a diagonal matrix if $\left|\lambda_{i}\right| \neq\left|\lambda_{j}\right|$.

Let

$$
u=P(x, z)
$$

be a transformation of the vector $u$ such that $P(x, z)$ is holomorphic for $\operatorname{Im}(x) \geqq R_{2},\|z\| \leqq \delta^{\prime \prime}$, and admits a uniformly asymptotic expansion

$$
P(x, z) \cong \sum_{k=0}^{\infty} x^{-k} P_{k}(z)
$$


for $\|z\| \leqq \delta^{\prime \prime}$ as $x$ tends to infinity in the region $\operatorname{Im}(x) \geqq R_{2}$, where $P_{k}(z)$ are holomorphic for $\|z\| \leqq \delta^{\prime \prime}$. The transformation $P$ will have a convergent representation in powers of $z$, which we assume has the form

$$
P(x, z)=z+\sum_{|\mathfrak{p}| \geqq 2} \hat{P}_{\mathfrak{p}}(x) z^{\mathfrak{p}} .
$$

Since $P$ admits the asymptotic expansion (1.6), the coefficients $\hat{P}_{p}(x)$ also admit asymptotic expansions in powers of $x^{-1}$.

We shall prove the following theorem:

ThEOREM. Let the eigenvalues $\lambda_{i}$ of $f_{0 y}(0)$ satisfy

$$
1<\left|\lambda_{1}\right|<\cdots<\left|\lambda_{n}\right|
$$

and

$$
\prod_{i=1}^{n}\left|\lambda_{i}\right|^{p_{i}} \neq\left|\lambda_{j}\right| \quad(j=1, \cdots, n,|\mathfrak{p}| \geqq 2) .
$$

Then there exists a transformation of the type (1.5) by which the system (1.4) is reduced to the linear system

$$
z(x+1)=B(x) z(x) .
$$

In order to determine the transformation $P$, we have to solve the functional equation

$$
P(x+1, B(x) z)=g(x, P(x, z)) .
$$

Let $Z(x)$ be a fundamental matrix of solutions for the linear system (1.10). Then the general solution of (1.10) can be written in the form

$$
z(x)=Z(x) c(x),
$$

where $c(x)$ is an arbitrary periodic vector-valued function of period 1 .

We shall show that if $x$ is restricted to a region of the form

$$
\operatorname{Im}(x) \geqq R_{2}, \quad \alpha \leqq \arg (x-a),
$$

where $\alpha$ is a positive constant determined by $\lambda_{1}, \cdots, \lambda_{n}$ and $a$ is an arbitrary real number, the fundamental solution $Z(x)$ is bounded and tends to zero as $x$ tends to infinity in the region (1.13). Hence, if $c(x)$ is sufficiently small the general solution of (1.1) is given by

$$
y(x)=p(x)+P(x, Z(x) c(x))
$$

in the region (1.13).

The scalar case, $n=1$, has been treated by J. Horn [5] under the assumption that $f(x, y)$ is holomorphic for $|x| \geqq R_{0},\|y\| \leqq \delta_{0}$ using Laplace transform techniques. W. J. Trjitzinsky [7] has treated the single $n$th order equation 


$$
y(x+n)=f(x, y(x), y(x+1), \cdots, y(x+n-1))
$$

under various assumptions including $f(x, 0,0, \cdots, 0) \equiv 0$, i.e., the existence of a solution $p(x) \equiv 0$. He constructed formal series expansions equivalent to our series (1.7) which he proved are asymptotic to true solutions, while we have established the convergence of this infinite series. Furthermore, our results, while extending known results such as these, are obtained in a simpler and more direct manner.

2. Formal solution in powers of $x^{-1}$. First of all, we shall determine a formal solution

$$
P(x, z)=\sum_{k=0}^{\infty} x^{-k} P_{k}(z)
$$

of the equation (1.11), so that $P_{k}(z)$ are holomorphic in a region

$$
\|z\| \leqq \delta^{\prime \prime} .
$$

By substituting (2.1) into (1.11) and identifying terms in both sides, we get

$$
P_{0}\left(B_{0} z\right)=g_{0}\left(P_{0}(z)\right) .
$$

This is Schröder's equation. Since

$$
g_{0}(y)=B_{0} y+O\left(\|y\|^{2}\right),
$$

and we assumed the conditions (1.8) and (1.9), there exists a solution

$$
P_{0}(z)=z+O\left(\|z\|^{2}\right)
$$

of (2.3) which is holomorphic in a region (2.2). (See M. Urabe [8].)

Put

$$
P(x, z)=P_{0}(z)+Q(x, z) .
$$

Then the equation (1.11) becomes

$$
Q(x+1, B(x) z)=F(x, z, Q(x, z)),
$$

where

$$
F(x, z, Q)=g\left(x, P_{0}(z)+Q\right)-P_{0}(B(x) z)
$$

Put

$$
Q(x, z)=\sum_{k=1}^{\infty} x^{-k} P_{k}(z)
$$

and 


$$
F(x, z, Q)=F_{0}(z, Q)+\sum_{k=1}^{\infty} x^{-k} F_{k}(z, Q) \text {. }
$$

Then

$$
F_{0}(z, Q)=g_{0}\left(P_{0}(z)+Q\right)-P_{0}\left(B_{0} z\right)=O(\|Q\|),
$$

since $P_{0}(z)$ is determined by (2.3). Put

$$
F_{0}(z, Q)=L(z) Q+O\left(\|Q\|^{2}\right) .
$$

Then

$$
L(z)=B_{0}+O(\|z\|) .
$$

On the other hand, since

$$
\begin{aligned}
F(x, z, 0) & =g\left(x, P_{0}(z)\right)-P_{0}(B(x) z)=B(x) P_{0}(z)+O\left(\left\|P_{0}(z)\right\|^{2}\right)-P_{0}(B(x) z) \\
& =B(x)\left\{z+O\left(\|z\|^{2}\right)\right\}-\left\{B(x) z+O\left(\|B(x) z\|^{2}\right)\right\}+O\left(\left\|P_{0}(z)\right\|^{2}\right) \\
& =O\left(\|z\|^{2}\right),
\end{aligned}
$$

we have

$$
F_{k}(z, 0)=O\left(\|z\|^{2}\right) \quad(k=0,1,2, \cdots) .
$$

By inserting (2.5) into (2.4) and identifying terms in both sides, we get

$$
P_{k}\left(B_{0} z\right)=L(z) P_{k}(z)+\mathfrak{S}_{k}(z) \quad(k=1,2, \cdots),
$$

where $\mathfrak{S}_{k}(z)$ is a polynomial of $P_{k^{\prime}}\left(k^{\prime}<k\right)$ and if $P_{k^{\prime}}\left(k^{\prime}<k\right)$ are $O\left(\|z\|^{2}\right)$, then $\mathfrak{S}_{k}(z)=O\left(\|z\|^{2}\right)$,. There exist $P_{k}(z)$ satisfying (2.7),

$$
P_{k}(z)=O\left(\|z\|^{2}\right)
$$

and holomorphic in a region (2.2). The region (2.2) is determined so that it is independent of $k$. In fact, let $\mathcal{D}$ be an open neighborhood of the origin such that

$$
B_{0}^{-1} \mathfrak{D} \subset \mathfrak{D}
$$

Because of the assumption (1.8), we can construct such a neighborhood in any neighborhood of the origin. Assume that a positive integer $N$ is so large that we have

$$
\|L(z)\|\left\|B_{0}^{-1}\right\|^{N} \leqq \frac{1}{2}
$$

for $z \in \mathfrak{D}$. Assume that $\mathfrak{S}_{k}(z)$ is holomorphic in $\mathfrak{D}$. Then there exists a holomorphic vector-valued function $P_{k}(z)$ such that

$$
P_{k}\left(B_{0} z\right)=L(z) P_{k}(z)+\subseteq_{k}(z)
$$

for $z \in \mathfrak{D}$. To prove this, first of all, construct a formal solution 


$$
P_{k}(z)=\sum_{|\mathfrak{p}| \geqq 0} P_{k \mathfrak{p}} z^{\mathfrak{p}} .
$$

It is possible to construct such a formal solution, because we have assumed (1.9). Put

$$
P_{k}^{(N)}(z)=\sum_{|p|=0}^{N-1} P_{k p} z^{p}
$$

and

$$
Q_{k}(z)=P_{k}(z)-P_{k}^{(N)}(z) .
$$

Then the equation (2.11) becomes

$$
Q_{k}\left(B_{0} z\right)=L(z) Q_{k}(z)+L(z) P_{k}^{(N)}(z)+\Im_{k}(z)-P_{k}^{(N)}\left(B_{0} z\right) .
$$

Put

$$
\mathfrak{T}_{k}(z)=L(z) P_{k}^{(N)}(z)+\mathfrak{S}_{k}(z)-P_{k}^{(N)}\left(B_{0} z\right) .
$$

Since (2.12) is a formal solution, we have

$$
\left\|\mathfrak{T}_{k}(z)\right\| \leqq M\|z\|^{N}
$$

for $z \in \mathfrak{D}$, where $M$ is a positive constant. Let $K$ be a positive constant such that

$$
K \geqq 2 M\left\|B_{0}^{-1}\right\|^{N} .
$$

Let $\mathfrak{F}$ be the set of all vector-valued functions $Q(z)$ such that

(i) $Q(z)$ is holomorphic in $\mathfrak{D}$,

(ii) $\|Q(z)\| \leqq K\|z\|^{N}$ in $\mathfrak{D}$.

$\mathfrak{F}$ is convex and compact with respect to the topology of uniform convergence on each compact subset of $\mathfrak{D}$. Define a transformation $T$ by

$$
T(Q)(z)=L\left(B_{0}^{-1} z\right) Q\left(B_{0}^{-1} z\right)+\mathfrak{T}_{k}\left(B_{0}^{-1} z\right)
$$

for $Q \in \mathfrak{F}$. Since we have (2.9), the right-hand member of (2.16) is holomorphic in $\mathfrak{D}$. Furthermore, (2.14) and the condition (ii) of $\mathfrak{F}$ imply

$$
\|T(Q)(z)\| \leqq\left\|L\left(B_{0}^{-1} z\right)\right\| K\left\|B_{0}^{-1}\right\|^{N}\|z\|^{N}+M\left\|B_{0}^{-1}\right\|^{N}\|z\|^{N}
$$

for $z \in \mathfrak{D}$. Since $B_{0}^{-1} z \in \mathfrak{D},(2.10)$ and (2.15) imply

$$
\|T(Q)(z)\| \leqq K\|z\|^{N}
$$

for $z \in \mathfrak{D}$. Thus we have $T(Q) \in \mathfrak{F}$. Therefore, there exists a fixed point of $T(Q)$ in $\mathfrak{F}$, because $T$ is continuous with respect to the topology specified above. This proves the existence of the solution of (2.13). The condition (2.8) is easily checked. Thus we can construct a formal solution of (1.11) in powers of $x^{-1}$. 
3. Transformation of equation (1.11). Let $\widetilde{P}(x, z)$ be an $n$-dimensional vector such that components of $\widetilde{P}$ are holomorphic for $\operatorname{Im}(x) \geqq R_{1},\|z\| \leqq \delta^{\prime \prime}$ and that $\widetilde{P}$ admits the uniformly asymptotic expansion

$$
\widetilde{P}(x, z) \cong \sum_{k=0}^{\infty} x^{-k} P_{k}(z)
$$

for $\|z\| \leqq \delta^{\prime \prime}$ as $x$ tends to infinity in the region $\operatorname{Im}(x) \geqq R_{1}$, where the right-hand member of (3.1) is the formal solution of (1.11). Furthermore, since we have $(2.8)$, we can assume that

$$
\widetilde{P}(x, z)=P_{0}(z)+O\left(\|z\|^{2}\right) .
$$

The existence of $\widetilde{P}$ is guaranteed by the Borel-Ritt Theorem [2].

Put

$$
P(x, z)=\widetilde{P}(x, z)+H(x, z) .
$$

Then the equation (1.11) becomes

$$
H(x+1, B(x) z)=G(x, z, H(x, z)),
$$

where

$$
G(x, z, H)=g(x, \widetilde{P}(x, z)+H)-\widetilde{P}(x+1, B(x) z) .
$$

The quantity $G(x, z, H)$ is holomorphic for

$$
\operatorname{Im}(x) \geqq R_{1}, \quad\|z\| \leqq \delta_{1},\|H\| \leqq \delta_{2} .
$$

Put

$$
G(x, z, H)=G_{0}(x, z)+G_{1}(x, z) H+\sum_{|\mathfrak{p}| \geqq 2} G_{\mathfrak{p}}(x, z) H^{\mathfrak{p}} .
$$

The coefficients $G_{0}, G_{1}$ and $G_{\mathfrak{p}}$ are holomorphic for $\operatorname{Im}(x) \geqq R_{1},\|z\| \leqq \delta_{1}$, and they admit asymptotic expansions in powers of $x^{-1}$. In particular, since

$$
G_{0}(x, z)=g(x, \widetilde{P}(x, z))-\widetilde{P}(x+1, B(x) z),
$$

we have

$$
G_{0}(x, z) \cong 0
$$

uniformly for $\|z\| \leqq \delta_{1}$ as $x$ tends to infinity in the region $\operatorname{Im}(x) \geqq R_{1}$, because the asymptotic expansion of $\widetilde{P}$ is the formal solution of (1.11). Furthermore, we have

$$
G_{0}(x, z)=O\left(\|z\|^{2}\right),
$$

because the forms of $g$ and $P_{0}$ and (3.2) imply 


$$
\begin{aligned}
G_{0}(x, z) & =B(x) \widetilde{P}(x, z)-P_{0}(B(x) z)+O\left(\|z\|^{2}\right) \\
& =B(x) P_{0}(z)-P_{0}(B(x) z)+O\left(\|z\|^{2}\right) \\
& =O\left(\|z\|^{2}\right) .
\end{aligned}
$$

On the other hand, we have

$$
G_{1}(x, z)=g_{u}(x, \widetilde{P}(x, z))=B(x)+O(\|z\|) .
$$

4. Lemmas on linear nonhomogeneous difference equations. In the sequel we shall need estimates of solutions of difference equations of the form

$$
y(x+1) a(x)=b(x) y(x)+f(x),
$$

where $a, b$ and $f$ are scalar functions which are assumed to be holomorphic and bounded for $\operatorname{Im}(x)>R_{1}$. Assume further that

$$
|a(x)| \geqq 1 / r, \quad|b(x)| \geqq 1 / r .
$$

for $\operatorname{Im}(x)>R_{1}$, where $r$ is a positive constant such that $r<1$. We also assume that either

$$
|a(x) / b(x)| \leqq r \text { for } \operatorname{Im}(x)>R_{1}
$$

or

$$
|b(x) / a(x)| \leqq r \text { for } \operatorname{Im}(x)>R_{1} .
$$

LEMma 1. There exists a holomorphic solution of the system (4.1) which satisfies the inequality

$$
|y(x)| \leqq \frac{1}{1-r} \sup _{\operatorname{Im}(x)>R_{1}}|f(x)|
$$

for $\operatorname{Im}(x)>R_{1}$.

Proof. Let the mapping $T$ be defined by

$$
T[y](x)=\frac{b(x-1)}{a(x-1)} y(x-1)+\frac{f(x-1)}{a(x-1)} \quad \text { if }\left(4.3^{\prime \prime}\right) \text { is satisfied, }
$$

and

$$
T[y](x)=\frac{a(x)}{b(x)} y(x)+\frac{f(x)}{b(x)} \quad \text { if }\left(4.3^{\prime}\right) \text { is satisfied. }
$$

A solution of (4.1) is equivalent to a fixed point of the mapping $T$.

Let $\mathfrak{F}$ be the set of all functions $y(x)$ holomorphic for $\operatorname{Im}(x)>R_{1}$ such that

$$
|y(x)| \leqq M
$$

where 


$$
M=\frac{1}{1-r} \sup _{\operatorname{Im}(x)>R_{1}}|f(x)| .
$$

Then, for $y \in \mathfrak{F}$, we have

$$
|T[y](x)| \leqq r M+r(1-r) M<M .
$$

Hence $T[y] \in \mathfrak{F}$.

$\mathfrak{F}$ is convex and compact with respect to the topology of uniform convergence on each compact subset of the region $\operatorname{Im}(x)>R_{1}$. Since the mapping $T$ is continuous with respect to the same topology, and $T[\mathfrak{F}]$ $\subset \mathfrak{F}$, there exists a member of $\mathfrak{F}$ such that

$$
y=T[y] \text {. }
$$

This is the desired solution, and the proof of Lemma 1 is completed.

REMARK. If $a, b$ and $f$ admit asymptotic expansions in powers of $x^{-1}$ as $x$ tends to infinity in the region $\operatorname{Im}(x)>R_{1}$, then the solution constructed above also admits an asymptotic expansion in powers of $x^{-1}$. This can be deduced from our previous results [3].

Let $b_{i}(x)(i=1,2, \cdots, n)$ be the diagonal elements of the diagonal matrix $B(x)$ which was given in the introduction. Then $b_{i}(x)$ admit asymptotic expansions

$$
b_{i}(x) \cong \lambda_{i}+\sum_{k=1}^{\infty} x^{-k} b_{i k}
$$

as $x$ tends to infinity in the region (1.3). Since we assumed (1.8) and (1.9), we have either

$$
\prod_{i=1}^{n}\left|b_{i}(x)\right|^{p_{i}}\left|b_{j}(x)\right|^{-1} \leqq r \quad \text { for }(1.3)
$$

or

$$
\left|b_{j}(x)\right| \prod_{i=1}^{n}\left|b_{i}(x)\right|^{-p_{i}} \leqq r \quad \text { for }(1.3)
$$

for $j=1,2, \cdots, n,|\mathfrak{p}| \geqq 2$, if $R_{1}$ is sufficiently large, where $r$ is a positive constant such that $r<1$. On the other hand, we can also have

$$
\left|b_{j}(x)\right| \geqq 1 / r \quad(j=1,2, \cdots, n)
$$

and

$$
\prod_{i=1}^{n}\left|b_{i}(x)\right|^{p_{i}} \geqq 1 / r \quad(|\mathfrak{p}| \geqq 2)
$$


for $(1.3)\left({ }^{3}\right)$.

Lemma 2. Assume that the scalar-valued function $f(x)$ is holomorphic and bounded for $\operatorname{Im}(x)>R_{1}$. Then there exists a holomorphic solution of

$$
y(x+1) \prod_{i=1}^{n} b_{i}(x)^{p_{i}}=b_{j}(x) y(x)+f(x)
$$

which satisfies the inequality

$$
|y(x)| \leqq \frac{1}{1-r} \sup _{\operatorname{Im}(x)>R_{1}}|f(x)| \quad \text { for } \operatorname{Im}(x)>R_{1} .
$$

This is a corollary of Lemma 1 . It is important that $r$ and $R_{1}$ are determined only by $B(x)$ and are independent of $j$ and $\mathfrak{p}$. If $f(x)$ admits an asymptotic expansion in powers of $x^{-1}$, then $y(x)$ also admits an asymptotic expansion in powers of $x^{-1}$.

5. Formal solution of equation (3.4). If we can construct a solution $H(x, z)$ of (3.4) such that

(i) $H(x, z)$ is holomorphic for $\operatorname{Im}(x) \geqq R_{2},\|z\| \leqq \delta_{3}$,

(ii) $H(x, z) \cong 0$ uniformly for $\|z\| \leqq \delta_{3}$ as $x$ tends to infinity in the region $\operatorname{Im}(x) \geqq R_{2}$,

(iii) $H(x, z)=O\left(\|z\|^{2}\right)$ for $\operatorname{Im}(x) \geqq R_{2},\|z\| \leqq \delta_{3}$, then we get the desired solution of (1.11) by defining $P$ by (3.3).

In order to construct such a solution $H$ of (3.4), first of all, we shall construct a formal solution of (3.4) in the following form:

$$
H(x, z)=\sum_{|p| \geqq 2} H_{p}(x) z^{\mathfrak{p}},
$$

where $H_{p}(x)$ are to be determined as holomorphic functions for $\operatorname{Im}(x)>R_{1}$. Inserting (5.1) into (3.4) we get

$$
\begin{aligned}
\sum_{|\mathfrak{p}| \geqq 2} & H_{\mathfrak{p}}(x+1)(B(x) z)^{\mathfrak{p}} \\
& =G_{0}(x, z)+G_{1}(x, z) \sum_{|\mathfrak{p}| \geqq 2} H_{\mathfrak{p}}(x) z^{\mathfrak{p}}+\sum_{|\mathfrak{p}| \geqq 2} G_{\mathfrak{p}}(x, z) H(x, z)^{\mathfrak{p}} .
\end{aligned}
$$

Since we have (3.9) and

$$
(B(x) z)^{\mathfrak{p}}=\prod_{i=1}^{n} b_{i}(x)^{p_{i}} z^{\mathfrak{p}}
$$

we have

( ${ }^{3}$ Case $\left(4.6^{\prime}\right)$ can occur at most a finite number of times since $\left|b_{j}(x)\right| \prod_{i=1}^{n}\left|b_{i}(x)\right|^{-p_{i}}$ $=O\left(r^{|\downarrow|}\right)$. 


$$
H_{\mathfrak{p}}(x+1) \prod_{i=1}^{n} b_{i}(x)^{p_{i}}=B(x) H_{\mathfrak{p}}(x)+h_{\mathfrak{p}}(x),
$$

where $h_{\mathfrak{p}}(x)$ is a polynomial of $P_{\mathfrak{p}^{\prime}}\left(\left|\mathfrak{p}^{\prime}\right|<|\mathfrak{p}|\right)$.

By the use of Lemma 2, we can determine $H_{p}$ successively. In particular, we have the estimates

$$
\left\|H_{\mathfrak{p}}(x)\right\| \leqq \frac{1}{1-r} \sup _{\operatorname{Im}(x)>R_{1}}\left\|h_{\mathfrak{p}}(x)\right\|
$$

for $\operatorname{Im}(x)>R_{1}$.

6. Majorant. In order to prove the convergence of the formal series (5.1), we shall construct a majorant.

Since $G_{0}(x, z)$ is asymptotically zero, for any positive integer $m$, there exists a positive constant $L_{m}$ such that

$$
\left\|G_{0}(x, z)\right\| \leqq L_{m}|x|^{-m}
$$

for

$$
\operatorname{Im}(x) \geqq R_{1}, \quad\|z\| \leqq \delta_{1} .
$$

On the other hand, there are positive constants $M_{1}$ and $M_{2}$ such that

$$
\left\|G_{1}(x, z)-B(x)\right\| \leqq M_{1}
$$

and

$$
\left\|G_{\mathfrak{p}}(x, z)\right\| \leqq M_{2} \delta_{2}^{-|\mathfrak{p}|}
$$

for (6.2). Put

$$
\begin{aligned}
& \phi_{0}(z)=\sum_{|\mathfrak{p}| \geqq 0} \delta_{1}^{-|\mathfrak{p}|} z^{\mathfrak{p}}, \\
& \phi_{1}(z)=\sum_{|\mathfrak{p}| \geqq 1} \delta_{1}^{-|\mathfrak{p}|} z^{\mathfrak{p}}
\end{aligned}
$$

and

$$
\phi_{2}(z)=\sum_{|\mathfrak{p}| \geqq 2} \delta_{1}^{-|\mathfrak{p}|} z^{\mathfrak{p}} .
$$

Then, since we have (3.8) and (3.9), $G_{0}(x, z)$ and $G_{1}(x, z)-B(x)$ are majorized respectively by $L_{m}|x|^{-m} \phi_{2}(z)$ and $M_{1} \phi_{1}(z)$, while $G_{\mathfrak{p}}(x, z)$ are majorized by $M_{2} \delta_{2}^{-|p|} \phi_{0}(z)$.

Consider the equation

$$
v=\frac{1}{1-r}\left\{\xi \phi_{2}(z)+M_{1} \phi_{1}(z) v+M_{2} \phi_{0}(z) \sum_{|\mathfrak{p}| \geqq 2} \delta_{2}^{-|\mathfrak{p}|} v^{|\mathfrak{p}|}\right\},
$$

where $v$ is a scalar and $\xi$ is a real nonnegative variable. 
The equation (6.5) has a solution of the form

$$
v=v(\xi, z)=\sum_{|\mathfrak{p}| \geqq 2} v_{\mathfrak{p}}(\xi) z^{\mathfrak{p}}
$$

which is holomorphic for

$$
|\xi| \leqq \rho, \quad\|z\| \leqq \delta_{3},
$$

if $\rho$ and $\delta_{3}$ are sufficiently small. It is easily seen that we have

$$
H_{\mathfrak{p}}(x) \leqq v_{\mathfrak{p}}\left(L_{m}|x|^{-m}\right)
$$

for $\operatorname{Im}(x)>\max \left\{\left(L_{m} / \rho\right)^{1 / m}, R_{1}\right\}$, because we have (5.4). Therefore, the series (5.1) converges uniformly in the largest such region

$$
\operatorname{Im}(x)>R_{2}^{\prime}, \quad\|z\| \leqq \delta_{3},
$$

where

$$
R_{2}^{\prime}=\max \left\{R_{1}, \inf _{m}\left(L_{m} / \rho\right)^{1 / m}\right\} .
$$

Furthermore, we have

$$
\|H(x, z)\| \leqq\left|v\left(L_{m}|x|^{-m}, z\right)\right|,
$$

and

$$
v(\xi, z)=O(\xi) .
$$

Hence $H(x, z) \cong 0$ uniformly for $\|z\| \leqq \delta_{3}$ as $x$ tends to infinity in the region $\operatorname{Im}(x)>R_{2}^{\prime}$ since $m$ is arbitrary. This completes the proof of our theorem.

REMARK. We can make $\rho$ arbitrarily large by making $\delta_{3}$ sufficiently small. Hence we can make $R_{2}^{\prime}=R_{1}$.

7. Estimates of fundamental matrix of solutions of (1.10). The system (1.10) is equivalent to $n$ scalar equations of the form

$$
w(x+1)=b(x) w(x),
$$

where $b(x)$ is holomorphic for $\operatorname{Im}(x) \geqq R_{2}$ and

$$
b(x) \cong \lambda+\sum_{k=1}^{\infty} x^{-k} c_{k}
$$

as $x$ tends to infinity in the region $\operatorname{Im}(x) \geqq R_{2}$, where $|\lambda|>1$. The function $b(x)$ can be written as

$$
b(x)=\lambda\left(1+x^{-1}\right)^{c_{1} / \lambda} \hat{b}(x),
$$

where $b(x)$ is holomorphic for $\operatorname{Im}(x) \geqq R_{2}$ and

$$
b(x)-1=O\left(|x|^{-2}\right)
$$


as $x$ tends to infinity in the region $\operatorname{Im}(x) \geqq R_{2}$. Then the product of solutions of three difference equations

$$
\begin{aligned}
& w_{1}(x+1)=\lambda w_{1}(x), \\
& w_{2}(x+1)=\left(1+x^{-1}\right)^{c_{1} / \lambda} w_{2}(x), \\
& w_{3}(x+1)=\hat{b}(x) w_{3}(x)
\end{aligned}
$$

is a solution of (7.1). The third equation of (7.4) is satisfied by

$$
\phi(x)=\prod_{m=1}^{\infty} \hat{b}(x-m) .
$$

Since we have (7.3), $\phi(x)$ is holomorphic and bounded for $\operatorname{Im}(x) \geqq R_{2}$. The first and the second equations of (7.4) are satisfied, respectively, by $\lambda^{(x-a)}$ and $x^{c_{1} / \lambda}$, where $a$ is an arbitrary real number. Thus we get a solution

$$
w(x)=\lambda^{(x-a)} x^{c_{1} / \lambda} \phi(x)
$$

of (7.1). Let $\theta=\arg (\log \lambda)$. Since $|\lambda|>1$, we have $|\theta|<\frac{1}{2} \pi$. The solution $w(x)$ is bounded for

$$
|\arg (x-a)+\theta-\pi| \leqq \frac{1}{2} \pi-\gamma, \quad \operatorname{Im}(x) \geqq R_{2},
$$

where $\gamma$ is an arbitrary positive number. Furthermore, $w(x)$ tends to zero as $x$ tends to infinity in the region (7.5).

For each of the scalar equations

$$
z(x+1)=b_{i}(x) z(x),
$$

results of this nature are available. The corresponding regions (7.5) have a nonempty intersection. Hence there exists a direction $\alpha$ such that the fundamental matrix $Z(x)$ of $(1.10)$ is bounded for (1.13) and tends to zero as $x$ tends to infinity in (1.13).

REMark. The region (1.13) is determined by the choice of $\log \lambda_{i}$.

8. General remarks. If the eigenvalues of $f_{0 y}(0)$ satisfy $0<\left|\lambda_{1}\right|<\left|\lambda_{2}\right|$ $<\cdots<\left|\lambda_{n}\right|<1$, similar results are available in a region of the form

$$
\operatorname{Im}(x) \geqq R, \quad \arg (x-a) \leqq \alpha<\pi .
$$

If the eigenvalues of $f_{0 y}(0)$ satisfy

$$
0<\left|\lambda_{1}\right|<\cdots<\left|\lambda_{k}\right|<1<\left|\lambda_{k+1}\right|<\cdots<\left|\lambda_{n}\right|,
$$

then similar results are available in the region $\operatorname{Im}(x) \geqq R, 0<\alpha \leqq \arg (x-a)$, if the first $k$ components of the vector $c(x)$ are chosen identically zero, while they are available in the region $\operatorname{Im}(x) \geqq R, \arg (x-a) \leqq \alpha<\pi$ if the last $n-k$ components of the vector $c(x)$ are chosen identically zero.

We note that the representations 


$$
g(x, u) \cong \sum_{k=0}^{\infty} x^{-k} g_{k}(u)
$$

and

$$
P(x, z) \cong \sum_{k=0}^{\infty} x^{-k} P_{k}(z)
$$

respectively, imply

$$
g(x, u)=B(x) u+\sum_{|\mathfrak{p}| \geqq 2} g_{\mathfrak{p}}(x) u^{\mathfrak{p}}
$$

and

$$
P(x, z)=z+\sum_{|\mathfrak{p}| \geqq 2} P_{\mathfrak{p}}(x) z^{\mathfrak{p}} .
$$

However, the converse is not true (see, Y. Sibuya [6]). If we only assume the representation (8.2) for $g$, we may directly construct $P$ in the form (1.7) in the same manner as in $\$ \$ 4,5$, and 6 .

A functional equation similar to (1.11) has been investigated by $R$. Bellman [1] in the study of stochastic transformations.

\section{BibLIOGRAPHY}

1. R. Bellman, Stochastic transformations and functional equations, IRE Trans. 7 (1962), 120.

2. K. O. Friedrichs, Special topics in analysis, Lecture notes, New York University, New York, 1953-1954.

3. W. A. Harris, Jr. and Y. Sibuya, Asymptotic solutions of systems of nonlinear difference equations, Arch. Rational Mech. Anal. 15 (1964), 377-395.

4. __ Note on linear difference equations, Bull. Amer. Math. Soc. 70 (1964), 123-127.

5. J. Horn, Úber eine nicht lineare Differenzengleichung, Jber. Deutsch. Math.-Verein. 26 (1918), 230-251.

6. Y. Sibuya, Asymptotic solutions of initial value problems of ordinary differential equations with a small parameter in the derivative. II, Arch. Rational Mech. Anal. 15 (1964), 247-262.

7. W. J. Trjitzinsky, Non-linear difference equations, Compositio Math. 5 (1937-38), 1-60.

8. M. Urabe, Equations of Schröder, J. Sci. Hiroshima Univ. Ser. A. 15 (1951), 113-131; 15 (1952), 203-233.

UNIVERSITY OF MiNNESOTA, Minneapolis, Minnesota

OCHANOMIZU UNIVERSITY, TOKYO, JAPAN 